\title{
Review article on hypersensitivity pneumonitis
}

\author{
Fatma A. Tony ${ }^{* 1}$, Youssef M. Soliman ${ }^{2}$ and Hoda A. Salem ${ }^{3,4}$
}

1 Department of Clinical Pharmacy Practice, Faculty of Pharmacy, British University in Egypt (BUE), Cairo, Egypt

2 Department of Chest, El-kasr AlAini, Giza, Egypt

3 Pharmacy Practice Department, Faculty of Pharmacy, Tabuk University

${ }^{4}$ Department of Clinical Pharmacy, Faculty of Pharmacy (Girls), Al-Azhar University, Cairo, Egypt..

* Correspondence: E-mail: Fatmatony242@gmail.com

\section{Article history: Received 05/06/2021 Revised 22/06/2021 Accepted 11/07/2021}

\begin{abstract}
Hypersensitivity pneumonitis (HP) which can also be called extrinsic allergic alveolitis is a condition that induces inflammation in the alveoli along with the terminal bronchioles. It mostly affects people after they inhale antigenic substances present in the surrounding environment. This reaction occurs in individuals who are genetically predisposed because of prolonged and frequent inhalation of different types of dusts, microorganisms, avian proteins, fungi, bacteria, and certain chemical compound of low molecular weight. Many factors are thought to affect the development and progression of HP, including the type and amount of the antigen, environmental conditions, antigen exposure frequency and intensity as well as interaction of immunity with offending antigens are all factors to consider. Like the majority of interstitial lung diseases, HP is a rare disorder. Both, incidence and prevalence of HP significantly differs worldwide, depending on the exposure to antigenic substances, geographical or local factors, cultural factors and genetic risk factors. The diagnostic criteria of HP rely mainly on a detailed history of exposure to antigen, clinical features, lung functions tests, High Resolution Computed Tomography (HRCT), laboratory tests and physical examination. Hypersensitivity Pneumonitis categorized as acute, subacute and chronic which varies in clinical features together with onset of symptoms. Corticosteroids are generally used in the treatment of HP, immunosuppressants and anti-fibrotic can be used in some HP cases. Lung transplantation is considered in cases where the disease has progressed to the end stages.
\end{abstract}

Keywords: Hypersensitivity Pneumonitis; Interstitial Lung Disease; Antigen Exposure; Fibrosis; High Resolution Computed Tomography.

This is an open access article distributed under the CC BY-NC-ND license https://creativecommons.org/licenses/by/4.0/

\section{INTRODUCTION}

Hypersensitivity pneumonitis (HP) is an interstitial lung disease. It is a complex syndrome, which differs in severity and clinical presentation ${ }^{\mathbf{1}}$. A variety of provocative elements have been discovered, including fungi, bacteria, mammalian or avian proteins, and some low molecular weight chemical compounds ${ }^{2}$. Bronchiolocentric granulomatous and cellular interstitial pneumonia characterize the disease, which primarily affects the terminal bronchioles, alveoli, and interstitium. Many factors are thought to affect the development and progression of HP including the type and amount of the antigen, environmental factors, antigen exposure frequency and intensity and finally the interaction of offending antigens with the immunity response ${ }^{3}$. The most susceptible occupational classes are those that work in areas contaminated by industrial organic dust from various sources, such as farmers or breeders.
HP can usually be easily reversed by detecting and eliminating the causative agents ${ }^{1}$. HP may be caused by a reaction to a single environmental agent or a combination of antigens inhaled. The incidence and prevalence of HP are still widely unknown ${ }^{4}$. Both, incidence and prevalence of HP differs worldwide, depending on the exposure to antigenic substances, geographical or local factors, cultural factors and genetic risk factors $\mathrm{HP}$ is categorized as acute, subacute and chronic which varies in clinical features together with onset of symptoms. Unfortunately, the clinicians found this classification hard to follow as there is no definite criteria to distinguish between these classes ${ }^{5}$. Diagnosis of HP depends on antigen exposure, pulmonary function tests, High Resolution Computed Tomography (HRCT), Broncho alveolar lavage (BAL), or biopsies ${ }^{3}$. 


\section{PATHOGENISIS}

Inhaled of antigens with diameter $5 \mathrm{~mm}$ or less could enter lung parenchyma where they deposit at respiratory bronchioles level after travelling through lymphatic vessels ${ }^{6}$. Exacerbated immune response that characterize HP may be induced by defective regulatory $\mathrm{T}$-cell activity or by increasing output of interleukin $-17^{3}$. There has been a proposal for a twohit hypothesis (existing genetic vulnerability or environmental factors plus the presence of inducing factors like antigens exposure), resulting in an exaggerated immune response which cause significant lung inflammation. Hypersensitivity lung damage is caused by both humoral and cellular processes $^{7}$.

Inflammation in acute episodes leads to triggering the immune-complexes, which may explain why symptoms don't appear until 4-8 hours after antigen exposure. This theory is encouraged by the existence of activated neutrophils, activated complement elements and increased neutrophils in BAL for acute HP patients, as well as those which were observed a few hours or days following antigen inhalation challenge ${ }^{8}$. Subacute and chronic HP is distinguished by a significant increase in $\mathrm{T}$ lymphocytes which marked by T-cell mediated response $^{9}$. T-cell alveolitis has no clear cause, but it is believed to be involved with an increase in T-cell recruitment and migration, decreased programmed cell death and increased proliferation in the surrounding environment ${ }^{4}$. Most people who are exposed to antigens develop immune tolerance, and antigen inhalation can cause a small increase in local lymphocytes with no clinical implications ${ }^{2}$. When genetic and environmental influences coexist, an exaggerated immune response develops, resulting in pronounced lung inflammation ${ }^{10}$.

\subsection{Sensitization}

Exposure of genetically predisposed individuals to antigens present in the environment can result in sensitization. The presence of unique antibodies in the serum, often $\mathrm{IgG}$, can be used to assess sensitization. Mostly, the sensitized individuals do not acquire HP but can occasionally have a slight elevation of BAL lymphocytes without clinical relevance ${ }^{11}$. Acute HP is thought to be caused by lung macrophage activation, which leads to the development of IL-1 plus TNF along with upregulation of adhesion molecules, which facilitate neutrophil adherence and migration towards interstitial lung spaces and alveolar spaces. ${ }^{12}$.

\subsection{Progression to fibrosis}

The mechanisms, conditions and processes that cause irreversible progression of fibrosis in HP remain unknown'. Epidemiological data indicate that particularly after 65 years, fibrotic HP rises with age. The mechanisms associated with aging are mainly due to irregular telomeres shrinkage and T-cells immune-senescence ${ }^{11}$. Age also is a factor that affects the presence of fibrotic characteristics associated with shorter survival ${ }^{3}$. Notably, gene clustering studies have shown that the fibrotic response is more powerful in middle-aged mice than in younger littermates, at least at the transcriptional level ${ }^{8}$. Furthermore, a reduced lifespan was attributed to an inability to recognize the inhaled antigen or continued exposure to a known antigen, most likely due to the development of fibrosis ${ }^{12}$.

\section{HISTOPATHOLOGY}

Despite there is little experience with acute HP, the morphology is characterized by lymphocytes, plasma cells, neutrophils, and macrophages infiltrating the interstitial space ${ }^{13}$. Amorphous proteinaceous exudates, edoema, or hemorrhage can be present in the alveolar spaces ${ }^{8}$. Bronchiolocentric interstitial granulomatous pneumonitis is the histologic characteristic of subacute and chronic $\mathrm{HP}^{9}$ as shown in figure 1. Depending on the HP form, variations are present in the granulomas ${ }^{3}$. Granulomas, for example, are small, coarsely differentiated, and loosely organized in the lungs of bird breeders ${ }^{14}$. Several patients presented with typical intra-alveolar buds made up from myofibroblasts and loose collagen-embedding fibroblasts, indicating organizing pneumonia ${ }^{15}$.Variable degrees of interstitial fibrosis characterize the chronic stage. HP can cause pulmonary fibrosis if there is mild to moderate lymphocyte infiltration, giant cells and sometimes poorly developed granulomas ${ }^{12}$. Researchers lately found that some HP patients would show up as the sole histopathological finding with interstitial pneumonitis with a non-specific pattern (NSIP) ${ }^{16}$. The most notable distinction is that NSIP has a uniform inflammatory or fibrotic lesion that occurs at the same time and in the same place, while HP has a distinctive bronchiolocentricity. Around 5\% to $10 \%$ of bird breeder's cases, their lung have shown this NSIP pattern ${ }^{17}$. The most prominent histologic features suggests lymphocytic hyperplasia and diffuse interstitial invasion of inflammatory cells ${ }^{8}$. 


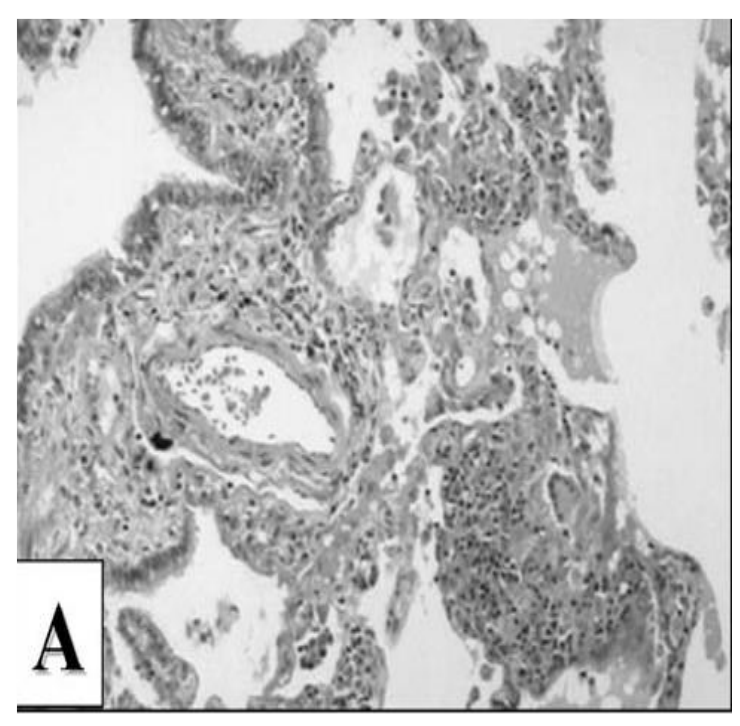

Figure 1. Granulomatous lesions in patients with subacute HP consist mostly of a cluster of giant cells and lymphocytes. There is also moderate interstitial lymphocytic infiltrate.

Modified from Costabel U, Miyazaki Y, Pardo A, Koschel $D$, Bonella F, Spagnolo P, et al 18

\section{PREVALENCE}

HP, like the majority of interstitial lung disorders is rare condition. ${ }^{15}$. Over the last two or three decades, analyses of the occurrence or prevalence of HP have highlighted the difficulties of researching the epidemiology of $\mathrm{HP}^{11}$. Due to methodological problems like study design and farmer lung hypersensitivity concept, such findings always been hard to identify ${ }^{15}$. Geographic factors that affect antigen exposure, such as climatic conditions and agricultural practices, make estimating HP incidence and prevalence much challenging ${ }^{9}$. Differences of HP and seropositivity between men and women are possibly due to differences in antigen exposure ${ }^{13}$. In general, genetic markers have been unable to confirm and validate inherited HP risk factors ${ }^{19}$.

\subsection{Cigarette smoking and hypersensitivity pneumonitis}

Nonsmokers are more likely than smokers to acquire HP, according to researches. Tobacco smokers presumed protective mechanisms which appears to be linked with immunosuppressive effect, mainly alveolar macrophages ${ }^{18}$. Acute cigarette smoke exposure can also cause alveolar macrophage apoptosis ${ }^{13}$. However, when the disease affects active smokers or who have recently quit, the disease has a more severe clinical path, and most patients have several episodes or a subtle onset, beside lower surviving rate than non-smokers ${ }^{20}$. Cigarette smoke also contains free radicals, which can exacerbate injury by causing structural protein damage in the lungs ${ }^{17}$

\section{DIAGNOSIS}

The key factor of the diagnosis of HP is obtaining a detailed history of exposure to antigen. Clinical presentation, lung function tests, HRCT, laboratory tests beside physical examination establish the diagnosis and evaluate the severity of the disease ${ }^{18}$. The most common lung function disorders, Like other interstitial lung diseases (ILD'S), the most frequent lung function abnormalities are ventilatory impairment and impaired gas ${ }^{3}$.

\subsection{Exposure}

HP has been linked to more than 200 antigens ${ }^{21}$. Antigens derived from fungal, protozoal, bacterial and small molecular weight chemical compounds are causative antigens ${ }^{16}$. Bacteria such as Thermophilic actinomycete found in moldy hay and straw, Klebsiella oxytoca found in humidifiers, mycobacteria such as Mycobacterium avium complex found in outdoor hot tubs ${ }^{22}$ and Mycobacterium immunogenum found in metal-working fluid ${ }^{23}$,fungi such as Absidia corymbifera found in moldy hay and straw and, Aspergillus fumigatus, Aspergillus Flavus found in moldy barley ${ }^{24}$ and other substances to which patient is sensitized commonly organic dusts of animals (i.e. raising birds) or vegetables, and rarely from chemicals ${ }^{4}$. Since most studies were focused on the workplace and antigens related environment, such as farmer's lung, suberosis, fish meal worker's lung and others, HP was previously classified as an occupational disorder ${ }^{20}$. Over many years, the home became the predominant causative environment over time, owing to individual cases of exposure to common antigens in everyday living conditions rather than evidence of major outbreaks ${ }^{23}$. Every year, new organic particle sources in the air are discovered.

\subsection{Imaging}

\subsubsection{Chest $x$-ray}

Conventional radiology showed poor sensitivity in patients with HP that have mild acute and subacute forms ${ }^{19}$. The acute type is characterized by transient diffuse or patchy ground-glass attenuation, as well as some locations of air space consolidation ${ }^{8}$. Fine nodular or reticulonodular shadowing with some ground-glass attenuation is shown clearly on the chest radiograph in subacute form ${ }^{24}$ as shown in Figure 2. 


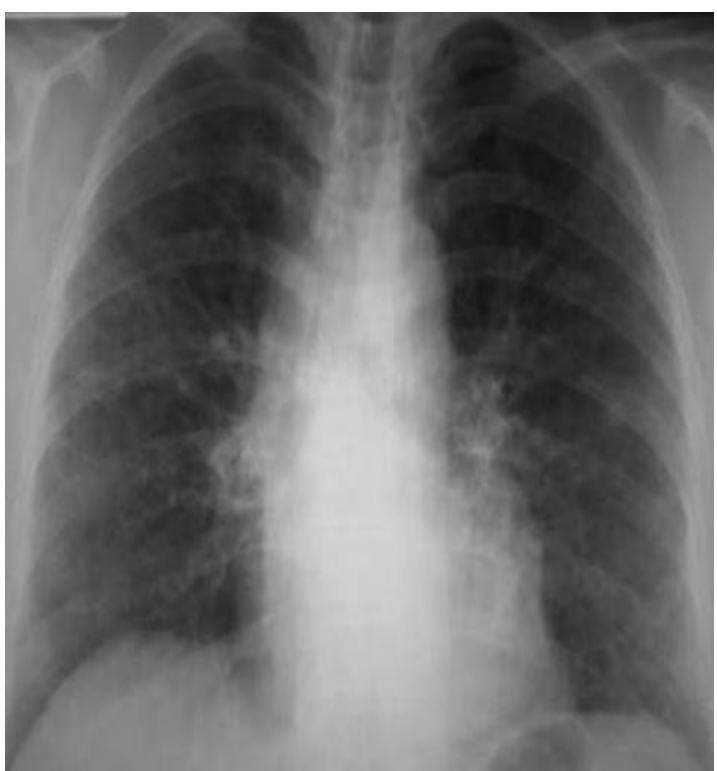

Figure 2. Chest radiograph of a patient with subacute HP shows a bilateral reticulonodular pattern.

Modified from "Selman M."24

\subsubsection{High resolution computed tomography (HRCT)}

HRCT was shown to have higher sensitivity than chest radiography for detecting $\mathrm{HP}^{25}$. The features of computed tomography are crucial in the diagnosis of $\mathrm{HP}^{18}$.

\subsubsection{Non-fibrotic HP}

The standard HP pattern is based on the detection of diffusely distributed HRCT findings, such as groundglass opacities (GGO's) as shown in Figure 3 and mosaic attenuation as shown in Figure 4, as well as at least one abnormality on HRCT, indicating small airway disease ${ }^{26}$. HRCT features of small airway disease on inspiratory images shows poor-defined, small $<5 \mathrm{~mm}$ centrilobular nodules as shown in Figure 5 and on expiratory images shows air trapping area as shown in Figure 6. Mosaic attenuation refers to coexisting areas with different attenuation in the lung parenchyma on inspiratory HRCT images ${ }^{27}$. Mosaic attenuation in nonfibrotic HP preferably reflects coexisting lobules with pneumonitis interspersed with lobules with regular or significantly decreased attenuation due to bronchiolar obstruction ${ }^{14}$. Although the presence of parenchymal cells abnormalities as well as symptoms of small airway disease strongly suggests nonfibrotic HP, isolated air trapping also can be seen in $\mathrm{HP}^{11}$.

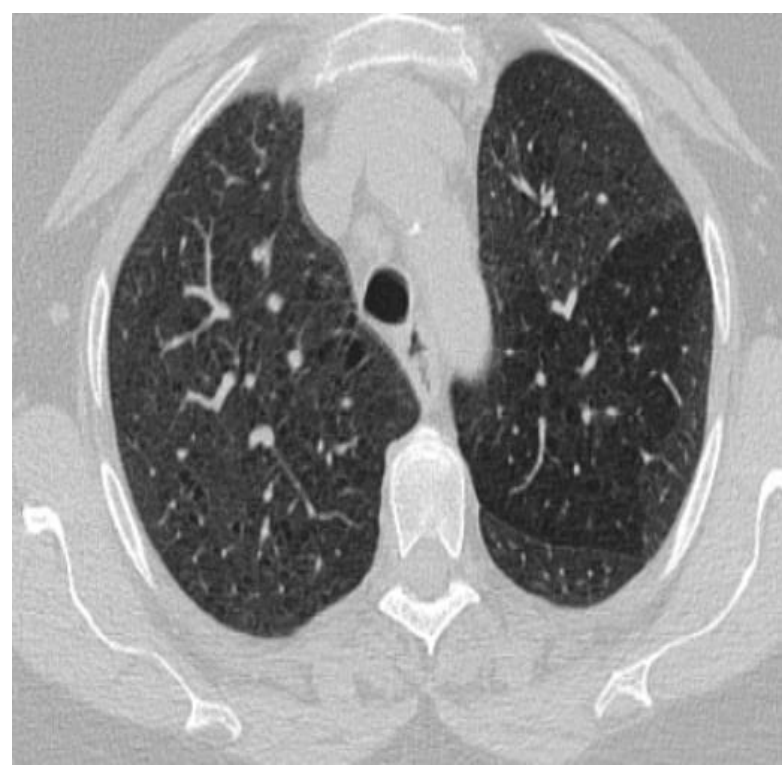

Figure 3. HCRT showing GGO'S

Modified from" Costabel U, Miyazaki Y, Pardo A, Koschel D, Bonella F, Spagnolo P, et al."

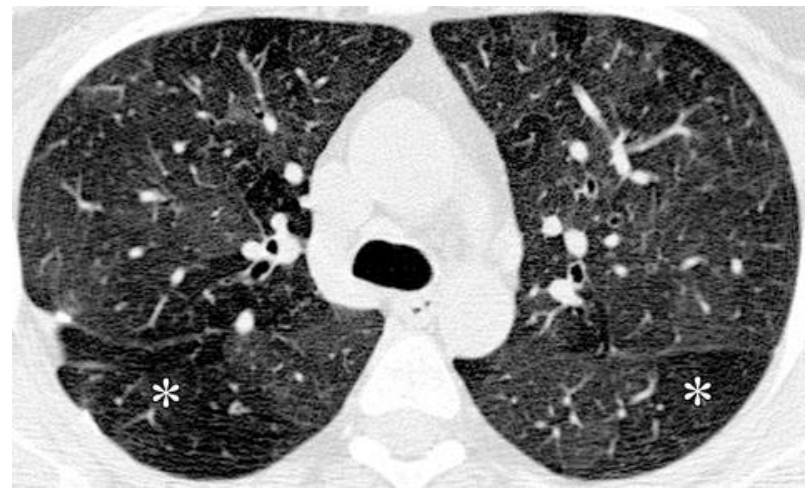

Figure 4. HCRT showing mosaic attenuation Modified from "Raghu G, Remy-Jardin M, Ryerson CJ, Myers JL, Kreuter M, Vasakova M, et al..” 28

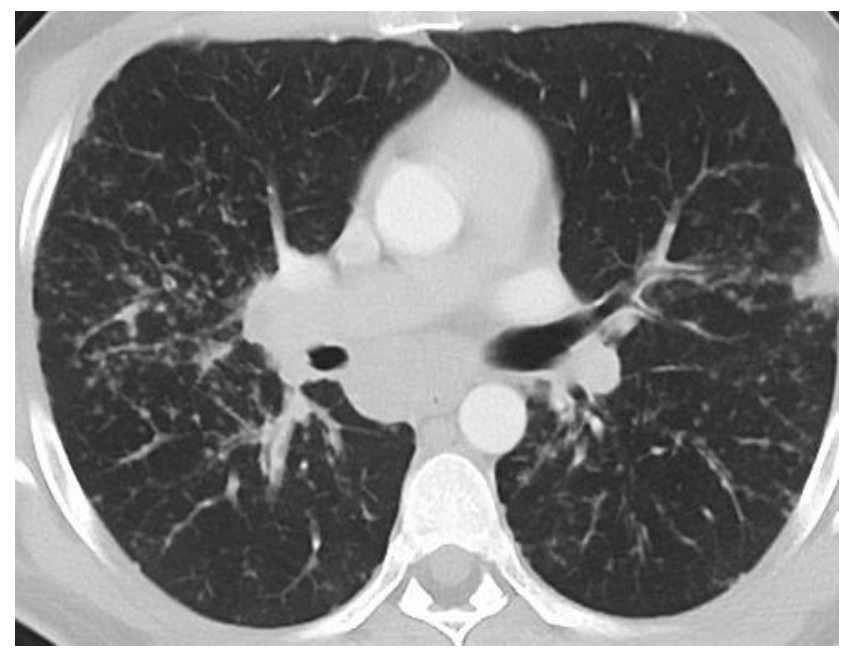

Figure 5. HCRT showing Centilobular Nodules Modified from "Mohr LC. “29 


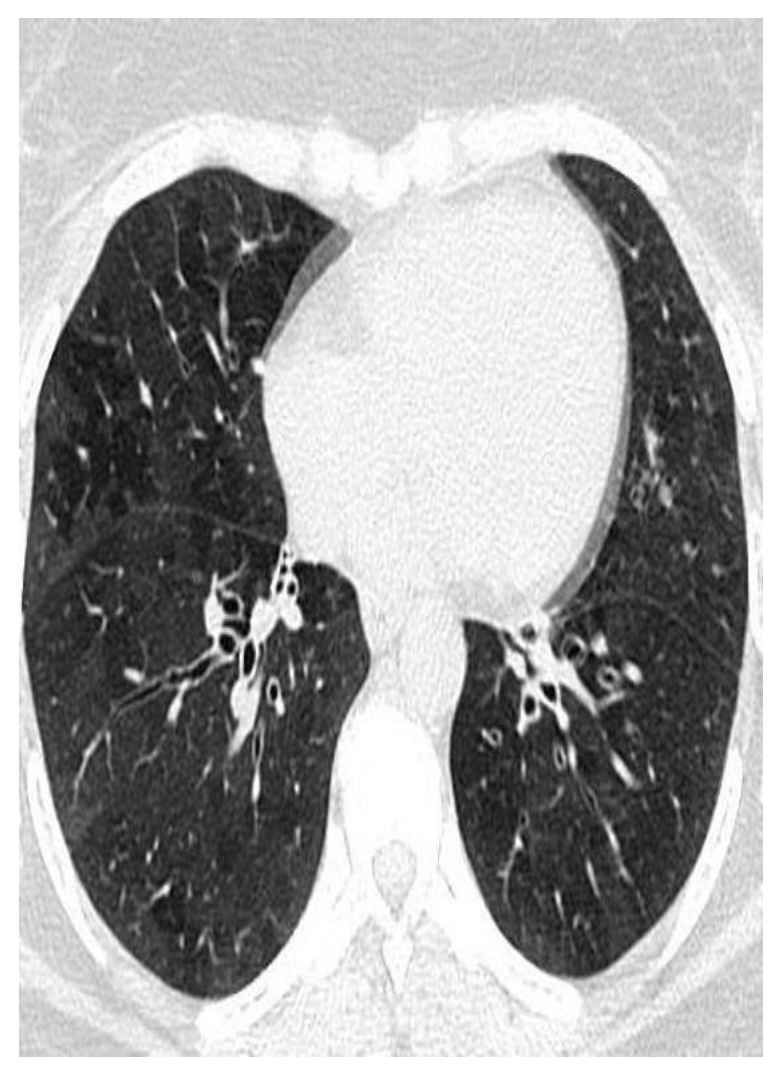

Figure 6. HCRT showing areas of air trapping Modified from "Raghu G, Remy-Jardin M, Ryerson CJ, Myers JL, Kreuter M, Vasakova M, et al.." 28

\subsubsection{Fibrotic HP}

Architectural lung distortion as abnormal fine or coarse reticulation, sometimes with septal thickening, is the most common symptom of HP lung fibrosis, that may appear alone or in conjunction with traction bronchiectasis in GGO's areas ${ }^{30}$. Lung fibrosis show the most severity in middle and lower zones of lung, or can be uniformly distributed in three lung zones with basal sparing ${ }^{31}$. On axial images, lung fibrosis does not have a central or peripheral predominance as shown in Figure 7.

In fibrotic HP, bronchiolar obstruction is manifested by many HRCT features ${ }^{32}$. While poorly established centrilobular nodules and mosaic attenuation can be seen in nonfibrotic $\mathrm{HP}^{33}$. Some variants are consistent with fibrotic HP in the distribution of fibrosis ${ }^{34}$, although they are less frequent. These involve fibrosis with a central or axially peripheral distribution, and basal-predominant disease ${ }^{31}$. Despite the fact that upper zone-predominant fibrosis has been identified as a distinguishing feature between fibrotic HP and idiopathic pulmonary fibrosis (IPF), upper lungpredominant disease affects only $10 \%$ of fibrotic HP patients ${ }^{35}$.

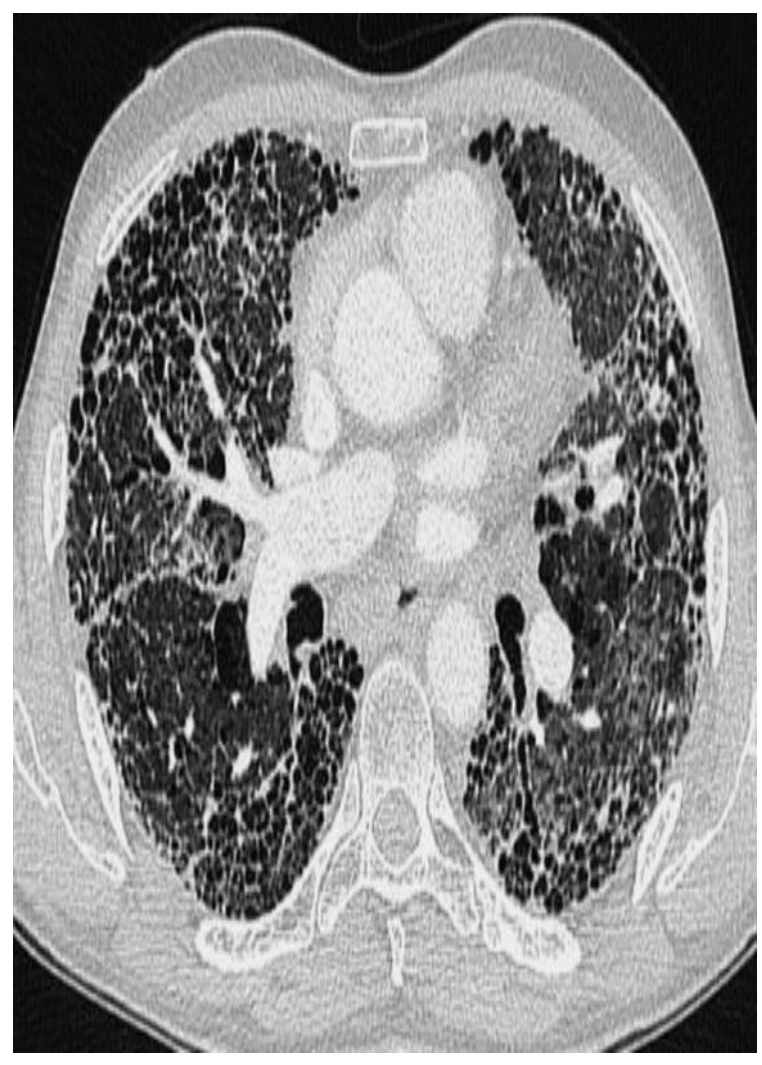

Figure 7. HCRT showing Fibrosis

Modified from" Costabel U, Miyazaki Y, Pardo A, Koschel D, Bonella F, Spagnolo P, et al."27

\subsection{Inhalation challenge}

For suspected settings, such as the workplace, inhalation challenges have been established as well as extensive provocation testing ${ }^{36}$. However, in these studies, there is no unified standard for protocols of inhalation or principles for determining a positive response $^{37}$. Additional research is needed before recommending inhalation challenge in the diagnosis of $\mathrm{HP}^{18 .}$

\subsection{Physiological features}

\subsubsection{Pulmonary function tests}

Lung function tests are characterized by a wide restriction is the respiratory pattern, which manifests a decreased forced vital capacity (FVC) and total lung capacity $^{24}$.The lung compliance static expiratory pressure-volume curve moves down and to the right of the standard curve, while lung recoil increases over the whole range of the decreased inspiratory capacity 34. At resting state, patients of mild to moderate severity may be presented with normal oxygen level in blood, but exercise can expose them to hypoxemia. In most cases, arterial $\mathrm{pH}$ is neutral ${ }^{38}$. 


\subsubsection{6-minute walk test (6MWT)}

The 6MWT considered to be good assessment of functional ability for people who have mild to severe impairment ${ }^{39}$. It has been widely used to evaluate the efficacy of pulmonary and cardiac disease clinical treatments. The American Thoracic Society released guidelines that provide a structured protocol for conducting the test ${ }^{40}$. The $6 \mathrm{MWT}$ was chosen over other walk tests because it is easier to administer, better tolerated, and better represents activities of daily life ${ }^{39}$. Pulse oximetry may also be used to measure arterial oxygen saturation as long as the oximeter, can be carried around, easily handled and lightweight ${ }^{41}$.

\subsection{Laboratory tests}

Elevated IgG antibodies aids in HP diagnosis also can promote detection of the responsible antigen source despite being only sensitization markers and antigen exposure and not disease indicators ${ }^{15}$. While there are various qualitative and quantitative commercial methods for detecting specific IgG antibodies, these methods vary in sensitivity and specificity and are available only for a small range of antigens ${ }^{42}$ IgG specific antibodies to cultured fungi and environmental gravity air culture can be tested simultaneously ${ }^{43}$. Specific $\operatorname{IgG}$ values of normal healthy individuals must be identified for quantitative methods, particularly for newly detected antigens Around half of healthy farmers express specific IgG antibodies to antigens found in the farming community, they show no symptoms of disease and have a prognosis comparable to those who do not have antibodies ${ }^{44}$.

\subsection{Bronchoalveolar lavage}

Other diagnostic techniques, such as BAL fluid analysis or biopsies of lung, could be very useful in the HP diagnosis ${ }^{43}$. The disease is characterized by significant rise in number of lymphocytes that account for more than $30 \%$ of recovered inflammatory cells in most cases and up to $50 \%$ in some cases ${ }^{6}$. However, as with the existence of specific antibodies, the existence of alveolar lymphocytosis may not rule out any possibility of a diagnosis with HP because asymptomatic, exposed individuals to antigen may show an increase in lymphocytes in $\mathrm{BAL}^{44}$. Infectious and non-infectious granulomatous diseases like sarcoidosis, miliary tuberculosis, and berylliosis all have almost comparable levels ${ }^{18 .}$

With a low CD4+/CD8+ ratio, a rise in $\mathrm{CD} 8+\mathrm{T}$ cells is reported by many researches. However, other researches have reported that in $\mathrm{CD} 8+\mathrm{T}$ cells can increase without ratio changing, Other studies have found a significant rise of CD4+ T cells along with an increase in $\mathrm{CD} 4+/ \mathrm{CD} 8+$ ratio $^{45}$. This variability can be explained by the involvement of many factors, like the form of HP, the clinical features, and the period between last antigen exposure along with obtaining BAL sample. Summer type HP differs significantly; which has a lower ratio of CD4+/CD8+, contrarily pigeon breeder disease, which has a higher CD4+/CD8+ ratio ${ }^{1}$.Chronic HP patients and smokers also display higher levels of CD4+. In conclusion, the ratio of $\mathrm{CD} 4+/ \mathrm{CD} 8+$ varies, there is no rule to estimate whether the ratio will increase or decrease ${ }^{45}$.

Differential diagnosis of HP is further expanded to exclude drug induced hypersensitivity syndrome which commonly manifests symptoms like dyspnea, cough or pleurisy ${ }^{10}$ or any drug toxicity with similar symptoms but the main aspect of diagnosis of HP is antigen exposures which differ HP from any drug induced toxicity with respiratory symptoms involvement $^{18}$

\section{CLINICAL BEHAVIOR AND MANAGEMENT}

HP clinical presentation is almost same regardless the type of the exposed antigen. Acute HP is described by the sudden onset of symptoms for a few hours following periodic and severe exposure to antigens ${ }^{16}$. Fever, dyspnea, chills, chest tightness, cough which could be dry or mild productive are all manifestations that appear in patients ${ }^{18}$. After removal of antigen exposure, within hours to days, symptoms improve, and clinical and radiographic results disappear entirely within weeks ${ }^{6}$. The subacute type can describe patients who have acute episodes with mild or absent respiratory symptoms, presenting as a nonspecific febrile condition before pulmonary symptoms appear ${ }^{21}$. Coughing and dyspnea are typical symptoms of subacute HP, which is a progressive disease in general $^{46}$. Different clinical manifestations of chronic HP may occur ${ }^{27}$. The clinical presentation of HP are diverse and determining the period of symptoms is difficult and can be highly variable. Although the duration or severity of symptoms have traditionally been used to differentiate between the different forms of HP; 
however, it is hard to distinguish between subacute and acute HP, so the term subacute is now eliminated and no longer used as a clinical subtype of $\mathrm{HP}^{44}$. Early diagnosis of HP, management, identifying and removing antigenic exposure is crucial ${ }^{1}$.

\subsection{Antigen exposure restriction}

To reduce the incidence of occupational risk it is necessary to follow some important instructions, including fluid control, enhancement of fresh air airing in addition to medical monitoring or limitation ${ }^{42}$. Preventive maintenance tests should be conducted regularly to assure that water systems, air conditioning, warming, and airing equipment are in good working order and that the indoor atmosphere is clean $^{24}$. Recurrence of the disease has been reported in all cases living in places that have not been disinfected from the provocative antigen or in homes where there has been inadequate cleaning ${ }^{17}$. The risk of HP in occupationally exposed individuals can be reduced by implementing current procedures and conditions that restrict antigen exposure ${ }^{36}$. Nevertheless, subsequent antigen avoidance in chronic fibrotic HP patients may not reverse the disease and some of them show gradual deterioration and ultimately die from the disease ${ }^{29}$.

\subsection{Pharmacological management}

\subsubsection{Corticosteroids}

Corticosteroids can be used as a pharmacological choice, without replacing the need to keep the patients away from exposure to antigens. Prednisolone is suggested in subacute and chronic cases, but their efficacy on long term must be assessed $^{39}$. Prednisolone at $0.5 \mathrm{mg}$ per $\mathrm{kg}$ daily for 8 weeks, succeeded by a step by step decrease until the maintenance dose is reached ${ }^{6}$. An example of the corticosteroids that are used in the treatment of HP is methylprednisolone which can regulate or prevent inflammation by reversing capillary permeability, preventing movement polymorphonuclear leukocytes and fibroblasts, regulating protein synthesis rate and stabilizing lysosomes at the cellular stage ${ }^{46}$.

Corticosteroids also inhibit cyclooxygenase (COX)-2 synthesis, which controls the synthesis of prostaglandins in damaged tissue, resulting in cascade inflammation ${ }^{6}$. Corticosteroids are linked to a variety of side effects, necessitating their tapering off or discontinuation as soon as the disease has been controlled $^{22}$. The common side effects include weight gain and obesity, nausea and vomiting, headache, swelling of ankles or feet or hands, confusion, increased thirst, restlessness, high blood pressure ${ }^{47}$. It also can cause serious adverse effects like allergic reactions, skin rashes and swelling of face, lips or tongue also may cause diabetes symptoms like increased thirst and urinate more often than normal ${ }^{46}$. Changes in emotions and mood, eye problems, infection, weakness and irregular heart rhythm are among the serious side effects that can be caused by corticosteroids.

Patients who have a proven hypersensitivity to corticosteroids or their components should avoid them ${ }^{46}$. Patients who suffer from osteoporosis, kidney failure, hypertension, heart problems, glaucoma, myasthenia gravis, peptic ulcers, ulcerative colitis or diabetes should use steroids with deep caution. Psychiatric symptoms such as euphoria, insomnia, personality changes, and depression can all occur when taking corticosteroids ${ }^{12}$. Corticosteroids should be used with caution in patients who are predisposed to psychosis, as psychotic symptoms can be intensified. $^{47}$

\subsubsection{Immunosuppressant}

The use of immunosuppressive therapy causes suppression in immune responses ${ }^{41}$. Immunosuppressive therapy was linked to increased mortality in cases who suffer from chronic fibrotic HP in retrospective longitudinal trial performed at four different tertiary medical centers in the United States $^{48}$. However, by comparing between the use of high-dose of prednisolone alone and with the use of prednisolone combined with azathioprine or mycophenolate mofetil (MMF), it was accompanied by the same risk of mortality plus urgency for lung transplantation or hospitalization ${ }^{49}$. The efficacy of MMF and azathioprine in the treatment of HP is still unknown ${ }^{48}$.

\subsubsection{Anti-fibrotic}

Additional anti-fibrotic treatment with nintedanib or pirfenidone can be considered in subjects with progressive HP despite conventional immunosuppressive therapy ${ }^{50}$. Other anti-fibrotic medications newly being screened for IPF may be considered for progressive fibrotic chronic $\mathrm{HP}^{51}$. Anti-fibrotic drugs have shown to delay the deterioration in patients' functional status and slow disease progression in IPF could be useful for 
progressive fibrotic chronic HP ${ }^{18}$. Nintedanib, a tyrosine kinase inhibitor, was shown to be effective in a recent trial that targets the receptor of fibroblast growth factor, receptor of vascular endothelial growth factor and receptor of platelet-derived growth ${ }^{52}$. It has been found that pirfenidone which is a drug with antifibrotic, antioxidant and anti-inflammatory properties, slows the declination rate of $\mathrm{FVC}^{34}$. Cases of progressive ILD fibrosis, as well as those with chronic fibrotic HP, can benefit from this treatment ${ }^{51}$.

\subsection{Lung transplantation}

There is no absolute effective treatment for progressive lung fibrosis which is reported in chronic progressive HP so lung transplantation is recommended during end-stages of the disease ${ }^{28}$. Survival rate after transplantation was determined in chronic HP then has been compared to IPF in a recent study $^{52}$. HP patients had significantly higher survival at 1,3 , and 5 years after lung transplantation (96 percent, 89 percent, and 89 percent versus 86 percent, 67 percent, and 49 percent) rather than in $\mathrm{IPF}^{52}$. HP patients showed decreased mortality risk in comparison with IPF patients ${ }^{24}$.

\section{COMPLICATIONS PROGNOSIS}

AND

The main complication of HP is the progression of fibrosis in the lung which is irreversible and leads increase the mortality rate of chronic HP patients ${ }^{27}$. Epidemiological data suggest that fibrosis increases with age, in particular after 65 years ${ }^{13}$. The prognosis of HP is widely varies depending on the type and duration exposure to antigens, the amount of antigen and the type of the disease. Generally, the acute form of HP seems to have a good prognosis ${ }^{53}$. After acute attacks, if the patient was diagnosed and treated rapidly, the patient in most cases experience complete remission $^{54}$. In chronic HP, the minimization of exposure to antigens is cirtical to avoid relapse and progression of fibrosis ${ }^{18}$.

\section{PREVENTION}

Education can prevent respiratory problems in highrisk environments ${ }^{55}$. Ideally, all farmers should be aware of the risks of barn dust exposure and encouraged to take effective preventive measures .Preventive measures may include mask wearing ,decrease contact with animals when possible, increasing ventilation ${ }^{1}$. However, for practical purposes, major preventive steps for primary prevention cannot be recommended and are typically reserved for people with previous HP history ${ }^{56}$.

Funding statement: This work is not funded.

Conflict of interest: The authors declare no conflict of interest.

Authors consent of publication: All authors give the Publisher the permission of the Authors to publish the Work.

Author Contribution: All Authors contributed equally in this work

\section{REFERENCES}

1. Sema M, Miyazaki Y, Tsutsui T, Tomita M, Eishi Y, Inase N. Environmental levels of avian antigen are relevant to the progression of chronic hypersensitivity pneumonitis during antigen avoidance: Levels of antigen and progression of chronic HP. Immun Inflamm Dis. 2018;6(1):15462 .

2.Lacasse Y, Selman M, Costabel U, Dalphin J-C, Morell F, Erkinjuntti-Pekkanen R, et al. Classification of hypersensitivity pneumonitis: a hypothesis. Int Arch Allergy Immunol. 2009;149(2):161-6.

3.Okamoto T, Miyazaki Y, Ogura T, Chida K, Kohno N, Kohno S, et al. Nationwide epidemiological survey of chronic hypersensitivity pneumonitis in Japan. Respir Investig. 2013;51(3):191-9.

4. Barnes H, Johannson KA. Management of fibrotic hypersensitivity pneumonitis. Clin Chest Med. 2021;42(2):311-9.

5. Soumagne $\mathrm{T}$, Chardon M-L, Dournes G, Laurent L, Degano B, Laurent F, et al. Emphysema in active farmer's lung disease. PLoS One. 2017;12(6):e0178263.

6. Vasakova M, Morell F, Walsh S, Leslie K, Raghu G. Hypersensitivity pneumonitis: Perspectives in diagnosis and management. Am $\mathbf{J}$ Respir Crit Care Med. 2017;196(6):680-9.

7. Girard M, Israël-Assayag E, Cormier Y. Impaired function of regulatory $\mathrm{T}$-cells in hypersensitivity pneumonitis. Eur Respir J. 2011;37(3):632-9.

8. Ishizuka M, Miyazaki Y, Inase N. Validation of new prediction score in inhalation provocation test 
for chronic hypersensitivity pneumonitis [abstract. Am J Respir Crit Care Med. 2017;195:A5412.

9. Barnes H, Morisset J, Molyneaux P, et al. A systematically derived exposure assessment instrument for chronic hypersensitivity pneumonitis. Chest 2020; 157:1506-1512.

10. Riario Sforza GG, Marinou A. Hypersensitivity pneumonitis: a complex lung disease. Clin Mol Allergy. 2017;15(1):6.

11. Adegunsoye A, Morisset J, Newton CA, Oldham JM, Vittinghoff E, Linderholm AL, et al. Leukocyte telomere length and mycophenolate therapy in chronic hypersensitivity pneumonitis. Eur Respir J. 2021;57(3):2002872.

12. Selman M, Lacasse Y, Pardo A, Cormier Y. Hypersensitivity pneumonitis caused by fungi. Proc Am Thorac Soc. 2010;7(3):229-36.

13. Fernandez Perez ER, Kong AM, Raimundo K, Koelsch TL, Kulkarni R, Cole AL. Epidemiology of hypersensitivity pneumonitis among an insured population in the United States: a claims-based cohort analysis. Ann Am Thorac Soc. 2018;15(4):460-9.

14. Tsutsui T, Miyazaki Y, Kuramochi J, Uchida $\mathrm{K}$, Eishi Y, Inase N. The amount of avian antigen in household dust predicts the prognosis of chronic bird-related hypersensitivity pneumonitis. Ann Am Thorac Soc. 2015;12(7):1013-21.

15. Almeida RF, Watte G, Marchiori E, Altmayer $\mathrm{S}$, Pacini GS, Barros MC, et al. High resolution computed tomography patterns in interstitial lung disease (ILD): prevalence and prognosis. J Bras Pneumol. 2020;46(5):e20190153.

16. Fernández Pérez ER, Kong AM, Raimundo K, Koelsch TL, Kulkarni R, Cole AL. Epidemiology of hypersensitivity pneumonitis among an insured population in the United States: a claims-based cohort analysis. Ann Am Thorac Soc 2018;15:460 469.

17. Sema M, Miyazaki Y, Tsutsui T, Tomita M, Eishi Y, Inase N. Environmental levels of avian antigen are relevant to the progression of chronic hypersensitivity pneumonitis during antigen avoidance: Levels of antigen and progression of chronic HP. Immun Inflamm Dis. 2018;6(1):15462.

18.Costabel U, Miyazaki Y, Pardo A, Koschel D, Bonella F, Spagnolo P, et al. Hypersensitivity pneumonitis. Nat Rev Dis Primers. 2020;6(1):65.https://www.nature.com/articles/s415 72-020-0191-z
19.Wälscher J, Gross B, Morisset J, Johannson KA, Vasakova M, Bruhwyler J, et al. Comorbidities and survival in patients with chronic hypersensitivity pneumonitis. Respir Res. 2020;21(1):12.

20. Kang J, Kim YJ, Choe J, Chae EJ, Song JW. Acute exacerbation of fibrotic hypersensitivity pneumonitis: incidence and outcomes. Respir Res. 2021;22(1):152.

21.Rittig AH, Hilberg O, Ibsen R, Løkke A. Incidence, comorbidity and survival rate of hypersensitivity pneumonitis: a national population-based study. ERJ Open Res. 2019;5(4):00259-2018.

22. Koster MA, Thomson CC, Collins BF, Jenkins AR, Ruminjo JK, Raghu G. Diagnosis of hypersensitivity pneumonitis in adults, 2020 clinical practice guideline: Summary for clinicians. Ann Am Thorac Soc. 2021;18(4):559-66.

23. Salisbury ML, Gu T, Murray S, Gross BH, Chughtai A, Sayyouh M, et al. Hypersensitivity pneumonitis: Radiologic phenotypes are associated with distinct survival time and pulmonary function trajectory. Chest. 2019;155(4):699-711.

24. Selman M. Hypersensitivity pneumonitis: a multifaceted deceiving disorder. Clin Chest Med. 2004;25(3):531-

47.https://www.sciencedirect.com/science/article/a bs/pii/S0272523104000450?via\%3Dihub

25.Wang P, Jones KD, Urisman A, Elicker BM, Urbania T, Johannson KA, et al. Pathologic findings and prognosis in a large prospective cohort of chronic hypersensitivity pneumonitis. Chest. 2017;152(3):502-9.

26. Hutchinson J, Hubbard R, Raghu G. Surgical lung biopsy for interstitial lung disease: when considered necessary, should these be done in larger and experienced centres only? Eur Respir J. 2019;53(2):1900023.

27. Morisset J, Johannson KA, Vittinghoff E, Aravena C, Elicker BM, Jones KD, et al. Use of mycophenolate mofetil or azathioprine for the management of chronic hypersensitivity pneumonitis. Chest. 2017;151(3):619-25.

28.Raghu G, Remy-Jardin M, Ryerson CJ, Myers JL, Kreuter M, Vasakova M, et al. Diagnosis of hypersensitivity pneumonitis in adults. An official ATS/JRS/ALAT clinical practice guideline. Am J Respir Crit Care Med. 2020;202(3):e36-69. https://www.atsjournals.org/doi/full/10.1164/rccm. 202005-2032ST. 
29. Mohr LC. Hypersensitivity pneumonitis. Curr Opin Pulm Med. 2004;10(5):401-11. https://journals.lww.com/copulmonarymedicine/fulltext/2004/09000/hypersens itivity_pneumonitis. 14

30. Walters GI, Mokhlis JM, Moore VC, Robertson AS, Burge GA, Bhomra PS, et al. Characteristics of hypersensitivity pneumonitis diagnosed by interstitial and occupational lung disease multi-disciplinary team consensus. Respir Med 2019;155:19-25.

31. Agache IO, Rogozea L. Management of hypersensivity pneumonitis. Clin Transl Allergy. 2013;3(1):1186 2045-7022-3-5.

32.Infante M, Lutman RF, Imparato S, Di Rocco M, Ceresoli GL, Torri V, et al. Differential diagnosis and management of focal ground-glass opacities. Eur Respir J. 2009;33(4):821-7.

33. Winstone T, Hague CJ, Churg A, Wright JL, Schellenberg R, Ryerson C. Biopsy-proven recurrent, acute, familial hypersensitivity pneumonitis: A case report and literature review. Respir Med Case Rep. 2018;24:173-5.

34. Quirce S, Vandenplas O, Campo P, Cruz MJ, de Blay F, Koschel D, et al. Occupational hypersensitivity pneumonitis: an EAACI position paper. Allergy. 2016;71(6):765-79.

35. Chung JH, Montner SM, Adegunsoye A, Oldham JM, Husain AN, Vij R, et al. CT findings associated with survival in chronic hypersensitivity pneumonitis. Eur Radiol. 2017;27(12):5127-35.

36.Barnett J, Molyneaux PL, Rawal B, Abdullah R, Hare SS, Vancheeswaran R, et al. Variable utility of mosaic attenuation to distinguish fibrotic hypersensitivity pneumonitis from idiopathic pulmonary fibrosis. Eur Respir J. 2019;54(1):1900531.

37.Tateishi T, Ohtani Y, Takemura T, Akashi T, Miyazaki Y, Inase N, et al. Serial high-resolution computed tomography findings of acute and chronic hypersensitivity pneumonitis induced by avian antigen. J Comput Assist Tomogr. 2011;35(2):2729.

38.Lacasse Y, Cormier Y. Hypersensitivity pneumonitis. Orphanet J Rare Dis. 2006;1(1):25.

39. Sema M, Miyazaki Y, Inase N. Measurement of avian antigen in household dust for management of chronic bird-related hypersensitivity pneumonitis [abstract. Am J Respir Crit Care Med. 2017;195:A1594.
40. Stavrou VT, Vavougios GD, Astara K, Siachpazidou DI, Papayianni E, Gourgoulianis KI. The 6-minute walk test and anthropometric characteristics as assessment tools in patients with obstructive sleep apnea syndrome. A preliminary report during the pandemic. J Pers Med. 2021;11(6):563.

41. Gimenez A, Storrer K, Kuranishi L, Soares MR, Ferreira RG, Pereira CAC. Change in FVC and survival in chronic fibrotic hypersensitivity pneumonitis. Thorax. 2018;73(4):391-2.

42. Ley B, Newton CA, Arnould I, Elicker BM, Henry TS, Vittinghoff E, et al. The MUC5B promoter polymorphism and telomere length in patients with chronic hypersensitivity pneumonitis: an observational cohort-control study. Lancet Respir Med. 2017;5(8):639-47.

43.Reboux G, Piarroux R, Roussel S, Millon L, Bardonnet K, Dalphin J-C. Assessment of four serological techniques in the immunological diagnosis of farmers' lung disease. J Med Microbiol. 2007;56(Pt 10):1317-21.

44. Raulf M, Joest M, Sander I, Hoffmeyer F, Nowak D, Ochmann U, et al. Update of reference values for $\mathrm{IgG}$ antibodies against typical antigens of hypersensitivity pneumonitis: Data of a German multicentre study. Allergo J. 2019;28(6):52-63.

45. Wanin S, Malka-Ruimy C, Deschildre A, Nathan N, Reboux G, Reix P, et al. Usefulness of bronchoalveolar lavage in a French pediatric cohort with hypersensitivity pneumonitis. Pediatr Pulmonol. 2020;55(1):136-40.

46. Li Y, Lian Z, Wang W, Niu Z, Ding W, Wu $\mathrm{C}$, et al. Establishment of rat models of different pathological types of hypersensitivity pneumonitis using pigeon droppings. Int Arch Allergy Immunol. 2021;1-9.

47. Paragliola RM, Papi G, Pontecorvi A, Corsello SM. Treatment with Synthetic Glucocorticoids and the Hypothalamus-Pituitary-Adrenal Axis". International Journal of Molecular Sciences. 2017;18(10):2201.

48.Adegunsoye A, Oldham JM, Fernández Pérez ER, Hamblin M, Patel N, Tener M, et al. Outcomes of immunosuppressive therapy in chronic hypersensitivity pneumonitis. ERJ Open Res. 2017;3(3):00016-2017.

49.Keir GJ, Maher TM, Ming D, Abdullah R, de Lauretis A, Wickremasinghe M, et al. Rituximab in severe, treatment-refractory interstitial lung 
disease: Rituximab in treatment-refractory ILD. Respirology. 2014;19(3):353-9.

51.Vasakova M, Selman M, Morell F, Sterclova M, Molina-Molina M, Raghu G. Hypersensitivity pneumonitis: Current concepts of pathogenesis and potential targets for treatment. Am J Respir Crit Care Med. 2019;200(3):301-8.

52. Maher TM, Corte TJ, Fischer A, Kreuter M, Lederer DJ, Molina-Molina M, et al. Pirfenidone in patients with unclassifiable progressive fibrosing interstitial lung disease: a double-blind, randomised, placebo-controlled, phase 2 trial. Lancet Respir Med. 2020;8(2):147-57.

53. Raimundo S, Pimenta AC, Cruz-Martins N, Rodrigues MC, Melo N, Mota PC, et al. Insights on chronic hypersensitivity pneumonitis' treatment: Factors associated with a favourable response to azathioprine. Life Sci. 2021;272(119274):119274.

54. Soumagne T, Chardon M-L, Dournes G, Laurent L, Degano B, Laurent F, et al. Emphysema in active farmer's lung disease. PLoS One. 2017;12(6):e0178263.

55. Wang P, Jones KD, Urisman A, Elicker BM, Urbania T, Johannson KA, et al. Pathologic findings and prognosis in a large prospective cohort of chronic hypersensitivity pneumonitis. Chest. 2017;152(3):502-9.

56. Singh S, Collins BF, Sharma BB, Joshi JM, Talwar D, Katiyar S, et al. Interstitial lung disease in India. Results of a prospective registry. Am J Respir Crit Care Med. 2017;195(6):801-13. 